\title{
Nonlinear desorption activation energy from TPD curves: analysis of the influence of initial values for the regression procedure
}

\author{
Carlo Pirola ${ }^{1}$, Alessandro Di Michele ${ }^{2}$ \\ ${ }^{1}$ Università degli Studi di Milano, Dipartimento di Chimica, via Golgi 19 - 20133 Milano (Italy) \\ ${ }^{3}$ Università degli Studi di Perugia, Dipartimento di Fisica e Geologia, via Pascoli-06123 \\ Perugia (Italy)
}

\footnotetext{
*To whom correspondence should be addressed

Tel: $++39 / 0250314283$

Fax: $++39 / 0250314300$

E-mail: carlo.pirola@unimi.it
} 


\section{Abstract}

Thermal Programmed Desorption (TPD) is a powerful technique for materials and catalysts characterization. By analysing TPD curves it is possible to calculate important parameters as the desorption activation energy $E_{d}$, that depends on the surface coverage $(\theta)$ by a non linear polynomial function, i.e. $E_{d}(\theta)=\sum_{k=0}^{N} \alpha_{k}(1-\theta)^{k}$.

The Polanyi-Wigner equation $-\frac{d \theta}{d T}=\left(\frac{A(\theta)}{\beta}\right) \theta^{n} \exp \left(\frac{-E_{d}(\theta)}{R T}\right)$ can be used as theoretical basis to calculate this parameter, by a fitting regression procedure starting from experimental TPD data. Different degrees (k) for this polynomial equation and different initial values of the frequency factor $A(\theta)$ were considered and discussed to obtain univocal value of desorption energy.

Three different Pt and Co based catalysts, suitable for hydrogenation reactions, have been considered as case studies for the application and validation of the proposed calculation procedure.

Keywords: TPD, Desorption Energy, Polanyi-Wigner equation, Regression, Initial values. 


\section{Introduction}

Temperature Programmed Desorption (TPD) is a well known technique for materials and heterogeneous catalysts characterization [1], usually coupled with Temperature Programmed Reduction (TPR) ${ }^{[2]}$ experiments. Several reviews discussed the usefulness of TPD and its principles ${ }^{[3,4]}$. Typically, in a TPD experiment we put a small amount of sample in a quartz reactor located in a furnace. The first operation is to flow the catalyst with an inert gas to clean the surface. Then a reacting gas is continuously sent to the reactor and it is adsorbed on the catalyst surface, depending on its chemico-physical properties. Subsequently, the temperature of the catalyst is increased using a carrier gas flow, with a temperature program $\beta(\mathrm{t})=\mathrm{dT} / \mathrm{dt}$ (with the temperature $\mathrm{T}$ usually being a linear function of the time $t$ ). The change of composition of the exiting gas, desorbed from the surface upon heating, is quantified with a TCD detector as a function of temperature. Thermal transient techniques can be used for the determination of detailed kinetics of adsorption, desorption and surface reaction phenomena $^{[5][6]}$.

Adsorption phenomena occur if the attractive interaction between a molecule and a surface overcomes the disordering effect due to thermal motion. Physisorption takes place when the attractive force is the result of van-der-Waals attractions and the dissociation energy is approximately $50 \mathrm{~kJ} / \mathrm{mol}^{[4]}$. Differently, chemisorption phenomena should be considered when chemical bonds are formed and they are characterised by dissociation energies greater than $50 \mathrm{~kJ} / \mathrm{mol}^{[4]}$. An important characteristic of molecular chemisorption is the dissociation of the adsorbed molecules due to the weakening of intramolecular bonds.

TPD curves can be interpreted for the determination of kinetic and thermodynamic parameters of desorption processes, as the frequency factor and desorption activation energy ${ }^{[7]}{ }^{[8]}$. An example of application of this concept is reported in Kanervo et al. ${ }^{[4]}$ where the combination of TPD experiments and kinetic analysis were used for studying microkinetics of heterogeneous porous catalysts.

Complex shape TPD curves and multiple peaks often characterize thermal gas desorptions of catalysts with heterogeneous surfaces, as metal oxides, polycrystalline metals ${ }^{[9]}$. These TPD diagrams are 
quite different respect the ones we can obtain from a homogeneous surface. The quantitative analysis of heterogeneous surfaces, important in all the practical catalytic applications, needs to consider as nonlinear the activation energy of desorption with the surface coverage ${ }^{[10]}$. In fact, these catalysts should be considered to have more complex energy distribution of surface sites and then a nonlinearity of the energy for the activation of desorption phenomena.

When a single adsorbed state is present and readsorption does not occur, the variation of the surface coverage $\theta$ with time $t$, i.e. the desorption rate, at constant value of the heating rate $(\beta)$, is given by the well known Polanyi-Wigner equation ${ }^{[11]}$, proposed in $1925^{[12]}$ :

$$
-\frac{d \theta}{d T}=\left(\frac{A(\theta)}{\beta}\right) \theta^{n} \exp \left(\frac{-E_{d}(\theta)}{R T}\right)
$$

where $A(\theta)$ is the frequency factor, $E_{d}(\theta)$ is the activation energy of desorption and $n$ the order of desorption. If we consider heterogeneous surfaces, both the frequency factor and the desorption activation energy are dependent on surface coverage. The order of desorption $n=1$ corresponds to a first order rate law. For $n=2$ (second order process) we consider for example recombinative desorption of diatomic molecules. Equation (1) is easily transformed in the one having $d \theta / d T$ as first member in the case of a linear variation of the temperature $T$ with time $\left(T=T_{o}+\beta t\right.$, then $\left.d t=d T / \beta\right)$. On the basis of equation (1), Tokoro et al. ${ }^{[10]}$ have developed a mathematical procedure which lets calculate the pre-exponential factor $A(\theta)$ and the parameters of the non linear function $E_{d}(\theta)$. One of advantages of Tokoro's method is the use of only one desorption run, instead of many desorption runs, each at a fixed value of the surface coverage $\theta^{[13]}$. This method is based essentially on the minimization of an objective function, which gives the square of the sum of the differences between the calculated and experimental values of $d \theta / d t$, or $d \theta / d T$, being the summation extended to all the values of $\theta$ in which the TPD curve $\theta v s . t$ is divided.

The function $E_{d}(\theta)$ decreases with the increase of $\theta$, therefore it can be written ${ }^{[10]}$ as:

$$
E_{d}(\theta)=\sum_{k=0}^{N} \alpha_{k}(1-\theta)^{k}
$$


where $\alpha_{k}$ values have the dimension of energy, for example $k J / m o l, k$ is an integer number and $N$ is an integer able to optimize the $E_{d}(\theta)$ function. The best value of $N$ is really one of the key points of Tokoro's method. In fact, it is indicated ${ }^{[10]}$ that "a large $N$ is deliverable, but if $N$ is too large it could give meaningless results", but no criteria have been indicated to avoid uncertainty in the results.

The novelty of the present paper is the discussion and analysis of a very particular case-study of regression based calculation, i.e. the right evaluation of desorption energy for heterogeneous surfaces, starting from experimental TPD data. The Polanyi-Wigner is an equation largely used but the robust regression of his parameters is not reported. The comparison of the results obtained by a robust or not robust regression procedure can be useful to quantify the advantage of an improved calculation procedure to obtain univocal parameters.

In another paper ${ }^{[14]}$, equation (1) was considered to evaluate the desorption energy distribution, using a complex numerical algorithm containing a key parameter $(\alpha)$ which, as $N$, must not be very low or very high; again such indication produces uncertainty in the results.

Falconer and Schwarz, discussing in their review ${ }^{[13]}$ the desorption and readsorption effects in the TPD experiments, derived the equation:

$$
-\frac{d \theta}{d T}=\frac{F}{\beta}\left(\frac{k_{d}(\theta) \theta^{n}}{F+V_{C} V_{m} k_{a}(1-\theta)^{P}}\right)
$$

where $F$ is the volumetric flow rate of the carrier gas, $V_{c}$ is the total solid volume, $k_{a}$ and $k_{d}$ the adsorption and desorption kinetic parameters, $n$ and $p$ the order of the desorption and adsorption, $V_{m}$ the number of surface sites per unit solid volume. Equation (3) reduces to equation (1) if $F \gg$ $V_{C} V_{m} k_{a}(1-\theta)^{P}$, i.e. if the readsorption is small. In this case the term $-\frac{d \theta}{d T}$ does not depend from the flow rate of the carrier gas. We have experimentally verified that for our experimental TPD measurements this is the case. In fact, changing $F$ from 10 to $30 \mathrm{ml} \mathrm{min}^{-1}$ both the shape and the temperature peak ( $\theta$ vs. time or $\theta$ vs. temperature) do not change significantly. Moreover, we have verified that internal diffusion of the desorbing gas is not rate limiting. This experiment has been made by using the catalyst having particles diameter in the range 105-149 $\mu \mathrm{m}$ (100- $140 \mathrm{mesh})$ and 
60-68 $\mu \mathrm{m}$ (220-240 mesh). The results show that no difference appears in the shape and temperature peak of TPD curve. Therefore we conclude that equation (1) is suitable to interpret our results. As discussed in detail by Luna et al. ${ }^{[15]}$, different approaches are reported in literature to obtain $E_{d}(\theta)$ by the Polanyi-Wigner equation. The easiest method considers this parameter as linear respect the coverage degree ${ }^{[16]}$ and the $E_{d}(\theta)$ can be calculated from the slope of $\ln \left(r_{\text {des }}\right)$ versus $1 / T$; this simplification, as already discussed, is not suitable for heterogeneous surfaces. More complex methods consider the desorption energy as non linear, and they can be obtained by fitting procedure with the experimental data of the TPD curve, using $E_{d}(\theta)$ as a fitting parameter. In this case, a value for the frequency factor $A(\theta)$ must be previously estimated ${ }^{[17]}$ or inserted in the regression procedure as second fitting parameter ${ }^{[18]}$.

The a priori estimation of $A(\theta)$ can be very difficult due to the great numerical differences possible for this parameter. On the other hand, numerical regression procedures can be very dependent by the numbers that we insert as initial values. In fact, an iterative procedure requires starting values that must be inserted in the first numerical cycle. Several methods and programs are reported in the literature for models analysis, which implements robust methods for parameter estimation of nonlinear models, able to detect outliers ${ }^{[19]}$. The influence of the initial values can be very critical for the final results, in particular in the applications where several minima of the error function can be found ${ }^{[20]}$. This is the case of the Polanyi-Wigner equation from TPD curves, as it will be discussed in the present paper. In fact, the aim of the work is to propose a new and easy calculation procedure in order to avoid the uncertainties in the determination of $E_{d}(\theta)$ and $A(\theta)$ values. The systematic study of the initial values for iterative calculation procedure is a well known topic in scientific literature, but it was not previously discussed for the correct and univocal determination of kinetic parameters of heterogeneous surface starting from TPD analysis.

Three different Pt or Co based metallic catalysts, suitable for hydrogenation reactions, have been considered as case studies for the application of the proposed numerical method. The first sample was a mixture $\mathrm{Pt} / \mathrm{Al}_{2} \mathrm{O}_{3}$ where $\mathrm{Pt}$ is present in a $3 \%$ in weight (named "Pt3-S"), as described and used in 
R. Giannantonio et al. ${ }^{[21]}$. The second sample is a mixture $\mathrm{Co} / \mathrm{SiO}_{2}$ where $\mathrm{Co}$ is present in a $10 \%$ in weight (named "Co10-1S") and the third was with the same composition but with a different kind of silica, discussed in the experimental section, and named "Co10-2S".

The first catalyst Pt3-S is useful to compare the values of $E_{d}(\theta)$ and $A(\theta)$ of the original work ${ }^{\text {[21] }}$ respect to the ones obtained in the present paper. The Co-based catalysts have been analysed using different TPD procedures and a comparison between weakly and strongly adsorbed hydrogen species peaks is proposed.

\section{Material and methods}

\subsection{Experimental}

Catalyst Pt3-S (BET surface $=77 \pm 2 \mathrm{~m}^{2} \mathrm{~g}^{-1}$ ) was prepared, characterized and analysed by TPD as described in R. Giannantonio et al. ${ }^{[21]}$. Briefly, thermal desorption analyses were performed in a high vacuum (base pressure $=10^{-6}$ torr) stainless steel apparatus provided with a particular system for gas introduction and equipped with a Thermo Fischer QTMD quadrupole mass spectrometer. More precisely we have considered curve D of figure 6 of such reference, referred to the sample of Pt3-S. Such data are referred to irreversible chemisorbed hydrogen. The adsorption temperature, i.e. the temperature at which hydrogen was adsorbed by this sample, was $305 \mathrm{~K}$.

Co $10 \%_{\text {wt }}$ was prepared using two completely different kinds of silica. For Co10-1S sample an Aldrich Product silica (BET surface $=504 \pm 2 \mathrm{~m}^{2} \mathrm{~g}^{-1}$, pore volume $0.75 \mathrm{~cm}^{3} \mathrm{~g}^{-1}$ ) has been used, while the second sample Co10-2S was supported on a particular aerogel silica (BET surface: $498 \pm 2 \mathrm{~m}^{2} \mathrm{~g}^{-1}$, pore volume $3.19 \mathrm{~cm}^{3} \mathrm{~g}^{-1}$ ). Both samples were prepared by impregnation using an aqueous solution of cobalt nitrate; before the impregnation the support was heated overnight at about $373 \mathrm{~K}$. The impregnation was performed in a Rotavapor apparatus at $313 \mathrm{~K}$, for $24 \mathrm{~h}$. After the impregnation, water was eliminated at $333 \mathrm{~K}$ in the same apparatus under moderate vacuum (base pressure $=200$ torr). The catalyst was then dried at $393 \mathrm{~K}$ in air and calcinated at $773 \mathrm{~K}$ for $4 \mathrm{~h}$ (heating rates $=30$ $\mathrm{K} \min ^{-1}$ ). 
Co10-1S sample has been analysed using a commercial apparatus (Thermoquest Mod. TPR/D/O 1100 instrument) equipped with a TCD detector. The prepared catalyst, after the calcination procedure was dried at $393 \mathrm{~K}$, then weighed and placed in a quartz reactor where it was fluxed with hydrogen $\left(30 \mathrm{ml} \mathrm{min}^{-1}\right)$, while the temperature was raised to $673 \mathrm{~K}$ at $10 \mathrm{~K} \mathrm{~min}^{-1}$ and then maintained at this temperature for sixteen hours, then it was fluxed with argon $\left(30 \mathrm{ml} \mathrm{min}^{-1}\right)$ at $673 \mathrm{~K}$ for 2 hours (heating rates $=30 \mathrm{~K} \mathrm{~min}^{-1}$ ). The sample was cooled at $398 \mathrm{~K}$ (adsorption temperature) and then treated with 15 hydrogen pulses $\left(0.3 \mathrm{~cm}^{3}\right.$ of $\mathrm{H}_{2}$ for each pulse) to perform hydrogen chemisorption, each pulse has been injected in a stream of helium as carrier gas. Finally, TPD analysis was performed in Ar flow (20 ml min$\left.{ }^{-1}\right)$ heating the sample from 398 to $673 \mathrm{~K}\left(30 \mathrm{~K} \mathrm{~min}^{-1}\right)$; after reaching the last temperature the samples were cooled in the same gas to room temperature. Co10-2S sample has been analysed according Bartholomew procedure ${ }^{[22]}$ using the same instrument. The sample was previously reduced in a quartz reactor in hydrogen at $673 \mathrm{~K}$ for $16 \mathrm{~h}$ and then loaded in the TPD reactor. The sample was cooled to the adsorption temperature of $193 \mathrm{~K}$ (dry ice/acetone) and the gas flow was switched from pure $\mathrm{H}_{2}$ to pure He. After calibrating the analyser with several $50 \mu$ pulses of $\mathrm{H}_{2}$, the temperature was raised to $623 \mathrm{~K}$ in flowing $\mathrm{He}$ (heating rates $=15 \mathrm{~K} \mathrm{~min}^{-1}$ ).

\subsection{Calculation procedure}

The function $\Phi$ to be minimized in order to calculate $E_{d}(\theta)$ and $A(\theta)$ parameters of equation (1) is:

$$
\Phi=\sum_{i=1}^{m}\left(C_{\text {exp }, i}-C_{c a l c, i}\right)^{2}
$$

where $C_{e x p, i}=\frac{d \theta_{i}}{d t_{i}}{ }^{[10]}$ and $C_{c a l c, i}$ is calculated for discrete value of $N$ from equations (1) and (2). The minimization has been performed using a MATLAB program (Version 6.0.0.88 Release 12).

Being $\Phi$ a function of $N+2$ parameters [i.e. $a_{k}(K=0 \rightarrow N)$ and $A(\theta)$, see equations (1) and (2)], to find the absolute minima is not a trivial question. Some conditions must be used to initialize the calculations. In fact, the starting values of the $N+2$ parameters strongly influence the achievement of 
a "relative" minimum which may not be the "absolute" one. We have verified that the value of $A(\theta)$ influences strongly the minimum of $\Phi$ function. This implies that different initial values of $A(\theta)$ must be given to the program in order to find the relative minima. The range $10^{0}<A(\theta)<10^{18}$, proposed in the following calculations, should be considered as sufficient to explore all the possible numerical solutions with a chemico-physical meaning. To our best knowledge, numerical values greater than the ones considered in this paper have never been reported for sample similar to the ones here discussed for desorption order equal to 1 or $2^{[15]}$. Then the optimization procedure has been performed, for each constant $A(\theta)$ parameter, by optimizing the coefficient $\alpha_{k}$ of the polynomial equation (2), i.e. $a_{0}+a_{1}\left(1-\theta_{m}\right)+\cdots+a_{k}\left(1-\theta_{m}\right)^{k}$ where $1<k<7$. The absolute minimum was therefore chosen among the "relative" ones. Being $N$ an integer, the best procedure is to fix $N$ (hence $N+1$ unknowns deriving from equation 2) and also a discrete, but wide series of $A$ values. For each $N$ and $A$ the minimum of $\Phi$ can be found using a minimization procedure.

\section{Results and discussion}

The physico-chemical characteristics of these catalysts, in particular dispersions, particle size and reduction degree influence the adsorption-desorption curves. The main characterization results of Pt3$\mathrm{S}$ are reported in in R. Giannantonio et al. ${ }^{[21]}$, while the characterization study for the CO10-1S sample is reported in V. Ragaini et al. ${ }^{[23]}$ and C. Pirola et al. ${ }^{[24]}$. Finally, the main characteristics of the sample Co10-2S are discussed by Bianchi et al. ${ }^{[25]}$. In particular, the metal dispersions for Pt3-S, Co10-1S and Co10-2S are 15.91\%, 1.83\% and 3.9\% respectively. The reduction of the metals on the different catalyst was performed by flowing hydrogen at $613 \mathrm{~K}$ for $16 \mathrm{~h}$ for the Pt based sample ${ }^{\text {[21] }}$ and at $673 \mathrm{~K}$ for $16 \mathrm{~h}$ for both the Co based catalysts. Using these conditions, the reduction degrees for all the catalysts are in the range $80-90 \%$.

The desorption peaks (chemisorbed hydrogen) for Pt3-S and Co10-1S samples are reported in figure 1a while the desorption peaks (physisorbed hydrogen and chemisorbed hydrogen) for Co10-2S sample are reported in figure $1 \mathrm{~b}$. 

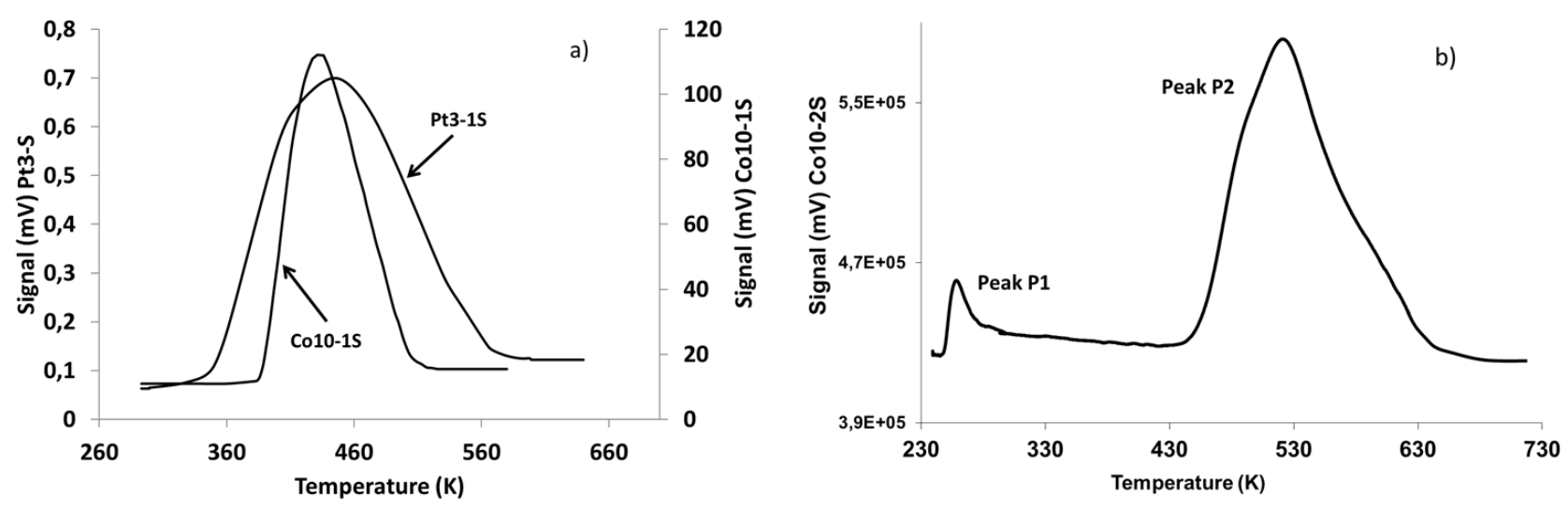

Figure 1: a) Hydrogen Thermal Desorption curves for the samples Pt3-S and Co10-1S; heating rate and adsorption temperature: Pt3-S: $5 \mathrm{~K} \mathrm{~min}^{-1}, T_{a d s}=305 \mathrm{~K}$; Col0-1S: $30 \mathrm{~K} \mathrm{~min}^{-1}, T_{a d s}=398 \mathrm{~K}$.

b) Hydrogen Thermal Desorption curves for the sample Co10-2S; heating rate and adsorption temperature: $30 \mathrm{~K} \mathrm{~min}^{-1}, T_{a d s}=193 \mathrm{~K}$.

The presence of two different TPD peaks in figure 1B is due to the experimental procedure ${ }^{[22]}$, applied to discriminate between weakly and strongly adsorbed hydrogen species. The higher temperature of the second peak respect the single peak of figure 1a can be dependent both on the different TPD procedures and on the different support-cobalt interactions present in Co- $1 \mathrm{~S}$ and Co- $2 \mathrm{~S}$ catalysts. In fact, the energy involved in the hydrogen reversibility is strongly dependent from several physical parameter (kind of support, dispersion, reduction degree) ${ }^{[22]}$ that are different in Co- $1 \mathrm{~S}^{[24]}$ and Co$2 \mathrm{~S}^{[25]}$ samples.

The numerical regression procedure previously described has been applied on these TPD data. The results for sample Pt3-S and for sample Co10-1S are shown in figure $2 \mathrm{a}$ and $2 \mathrm{~b}$ respectively, with $N$ varied from 2 to 7 and $A(\theta)$ from 1 to $10^{14}$. In these figures, the error function given by the equation (4) is reported vs. the different values of the frequency factor $(A(\theta)$ in equation (1)) used to develop the regression procedure. The different curves corresponds to different degrees of the polynomial 
equation (2) used for the different regressions. It is possible to observe the presence of several mathematical minima of the function error, but only the right combination of initial value of frequency factor and polynomial degree gives the univocal solution with chemical-physical meaning.
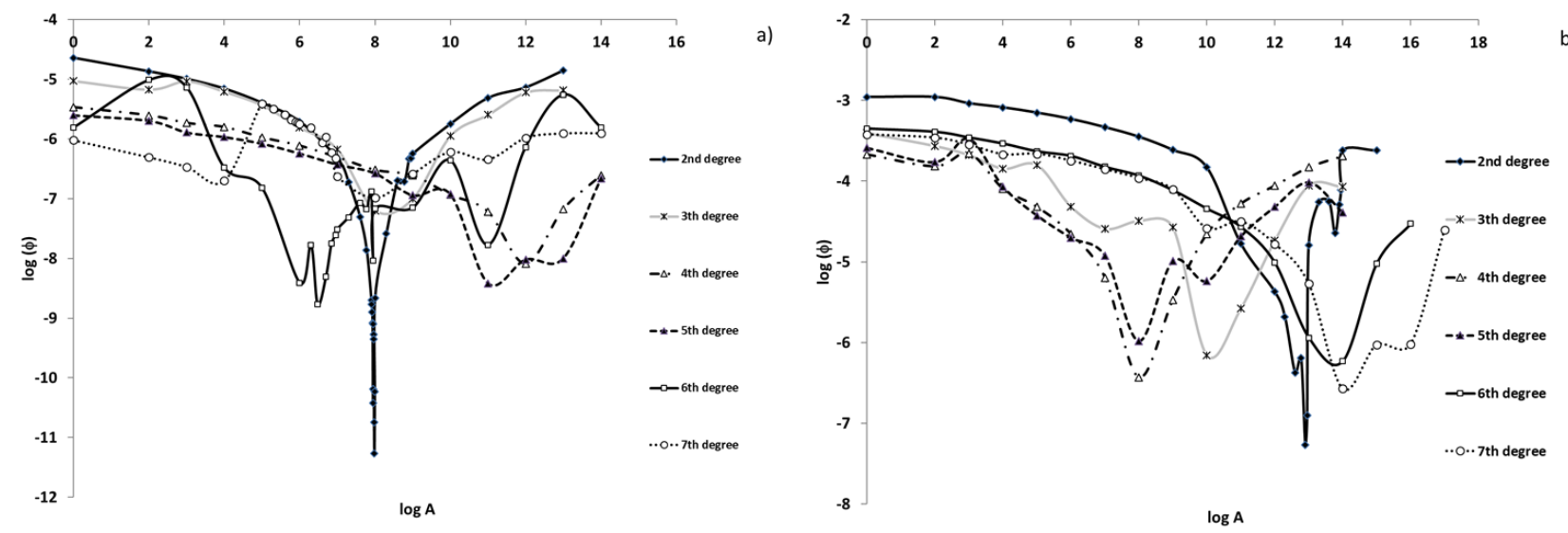

Figure 2: $\Phi$ function (equation 4) for the optimization procedure using different $A(\theta)$ values for the start of calculations and different $N$ degree (from 2 to 7 ) of equation (2) for Pt3-S sample (a) and Co10-1S sample (b). Logarithmic scale.

The couple of parameters $N=2$ and $A(\theta)=9.6 \times 10^{7} \mathrm{~s}^{-1}$ give the absolute minimum of $\Phi$ for the platinum based sample while for the cobalt based one the best result is obtained with $N=2$ and $A(\theta)=$ $9.0 \times 10^{12} \mathrm{~s}^{-1}$. Besides, where $N \geq 4$ the curves of $\Phi$ have more than one minima, each at different value of $A(\theta)$. The corresponding polynomial equations $E_{d}(\theta)$ as function of $\theta$, for each $N$ value, are shown graphically in figure 3. For the best value of $N(N=2)$ the polynomial is: for Pt3-S sample $E_{d}(\theta)=16.36+5.96(1-\theta)-3.02(1-\theta)^{2}$ and for Co10-1S sample $E_{d}(\theta)=25.05+$ $2.29(1-\theta)-0.55(1-\theta)^{2}$. These results are summarized in table 1 . 
a)
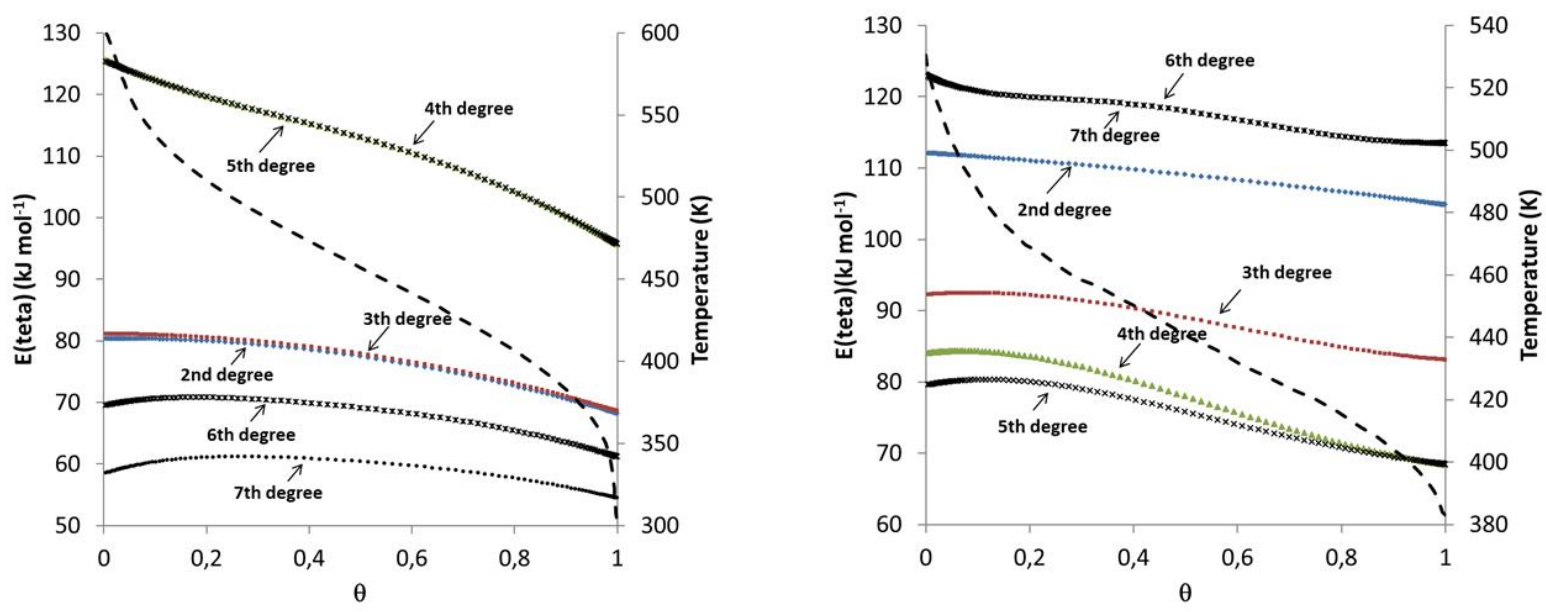

Figure 3: $E_{d}(\theta)$ calculated using the best-optimized parameters (i.e. the parameters by which the lowest $\Phi$ function has been calculated) for each different degree $N$ of polynomial of equation (2) for Pt3-S sample (a) and Co10-1S sample (b). Dashed line represents temperature $(K)$.

Table 1: Calculated values of Desorption energy $E_{d}(\theta), \mathrm{kJ} \mathrm{mol}^{-1}$, pre exponential factor $A(\theta)\left(s^{-1}\right)$ and optimized $N$ parameter for equation (2).

\begin{tabular}{|c|c|c|c|c|}
\hline Sample & $E_{d}(\theta \sim 0)$ & $E_{d}(\theta=1)$ & $A(\theta)$ & 2 \\
\hline Pt3-S & 80.4 & 68.2 & $9.02 \times 10^{12}$ & 2 \\
\hline Co10-1S $(n=2)$ & 112.2 & 80.4 & $9.6 \times 10^{7}$ & 4 \\
\hline Co10-2S & 24.8 & 35.4 & $1 \times 10^{6}$ & 3 \\
\hline TPD peak P1, $n=1)$ & & & $1 \times 10^{14}$ & 3 \\
\hline Co10-2S & 65 & 75 & $1 \times 10^{18}$ & \\
\hline Co10-2S & 216.8 & 175.7 & & \\
\hline
\end{tabular}

For each different value of $N$, the energy of desorption $E_{d}(\theta)$ has been calculated from equation (2). The results of both catalysts are reported in figure 3 where for $N=2$ (which leads to absolute minima 
of $\Phi)$ the $E_{d}(\theta)$ values are between $80.4 \mathrm{~kJ} \mathrm{~mol}^{-1}(\theta=0)$ and $68.2 \mathrm{~kJ} \mathrm{~mol}^{-1}(\theta=1)$ for Pt based catalyst and between $112.2 \mathrm{~kJ} \mathrm{~mol}^{-1}(\theta=0)$ and $104.7 \mathrm{~kJ} \mathrm{~mol}^{-1}(\theta=1)$ for Co based catalyst. From these results, it is possible to conclude that there are interactions between adjacent molecules on sites, which confirms that the surface energies depend on the sites and influences the adsorption energy.

Such results for Pt-based catalyst are close to those obtained experimentally by Procop and Volter and by Lisowsky who studied the adsorption-desorption of hydrogen on platinum foils, obtaining an average value of $E_{d}(\theta)$ of $69.5 \mathrm{~kJ} \mathrm{~mol}^{-1}[26]$ and from 51.5 to $103.4 \mathrm{~kJ} \mathrm{~mol}^{-1}{ }^{[27]}$. For what concerns the pre-exponential factor $A(\theta)$, in the literature such value is given frequently in the unit $v m^{2}$. $\left(H_{2} \text { molecules } \cdot s\right)^{-1}{ }^{[28]}$. To convert such unit in $s^{-1}$, as used in our calculations, it is necessary to multiply such unit by the factor $\mathrm{H}_{2}$ adsorbed molecules $\cdot(\mathrm{cm} \text { of adsorbing surface })^{-2}$. Such value ranges from $1 \times 10^{14}, 6 \times 10^{14}{ }^{[29]},<2.5 \times 10^{14}{ }^{[26]}$ considering polycrystalline $\mathrm{Pt}$ and a stoichiometry $1 \mathrm{H}_{2} / 2 \mathrm{Pt}$. Assuming an average value of $3 \times 10^{14}$ it derives that the values of $A(\theta)$ reported in literature are (in $\mathrm{s}^{-1}$ ): $1.6 \times 10^{6}, 4.5 \times 10^{7}, 3.4 \times 10^{6}{ }^{[28]}$ and $4.0 \times 10^{7}{ }^{[26]}$. Such values are close to the one above calculated $\left(9.6 \times 10^{7} \mathrm{~s}^{-1}\right)$ taking also into account both the different situations of $\mathrm{Pt}$ surface and the adsorption techniques among references.

The desorption energy and the pre-exponential factor for the Pt3-S catalyst were calculated in a previous paper ${ }^{[21]}$, by a similar fitting procedure based on the Polanyi-Wigner equation. Nevertheless, in this paper the range of $A(\theta)$ values was limited between $10^{12}$ and $10^{14} \mathrm{~s}^{-1}$. Moreover, a polynomial of fifth degree was used for the description of the desorption energy function, i.e. inserting $N=5$ in equation (2). In this work, the optimum value of $A(\theta)$ of $1-5 \times 10^{13} \mathrm{~s}^{-1}$ and values of $E_{d}(\theta)$ from 92.1 to $150.7 \mathrm{~kJ} \mathrm{~mol}^{-1}$ were found. However, the minimum of $\Phi$ function using such parameters (see figure 2) is not an absolute one, corresponding instead, as previously reported, to $A(\theta)=9.6 \times 10^{7} \mathrm{~s}^{-1}, N=2$ giving $68.2<E_{d}(\theta)<80.4 \mathrm{~kJ} \mathrm{~mol}^{-1}$.

Concerning the cobalt based catalyst, a comparison can be made considering the average value of desorption energy of $168 \mathrm{~kJ} \mathrm{~mol}^{-1}$ reported by in J. Zowtiak ${ }^{[30]}$ using the method of elaboration of 
TPD proposed in J. L. Falconer and J. A. Schwarz ${ }^{[13]}$. Zowtiak's results show that $E_{d}(\theta)$ changes both with the percentage of cobalt and with the type of support (i.e. for $\mathrm{Co} 10 \%$ wt $/ \mathrm{Al}_{2} \mathrm{O}_{3}, E_{d}(\theta)=144$ $\mathrm{kJ} \mathrm{mol}^{-1}$ ). Our silica support is different from that used in $\mathrm{J}_{\text {. Zowtiak }}{ }^{[30]}$, therefore $E_{d}(\theta)$ results in table 1 for Co10-1S are only qualitatively comparable with the average value above reported.

The values so found of $A(\theta)$ and $E_{d}(\theta)$ functions are very useful to calculate through equation (2) the time necessary for a change in the coverage $\Delta \theta$. The results for Pt3-S and Co10-1S samples are shown in figure 4, using the optimized results above reported. According to such results the desorption of hydrogen from cobalt based catalyst is much more rapid than that from Pt catalyst as expected from the large difference with calculated valued of the desorption constants.
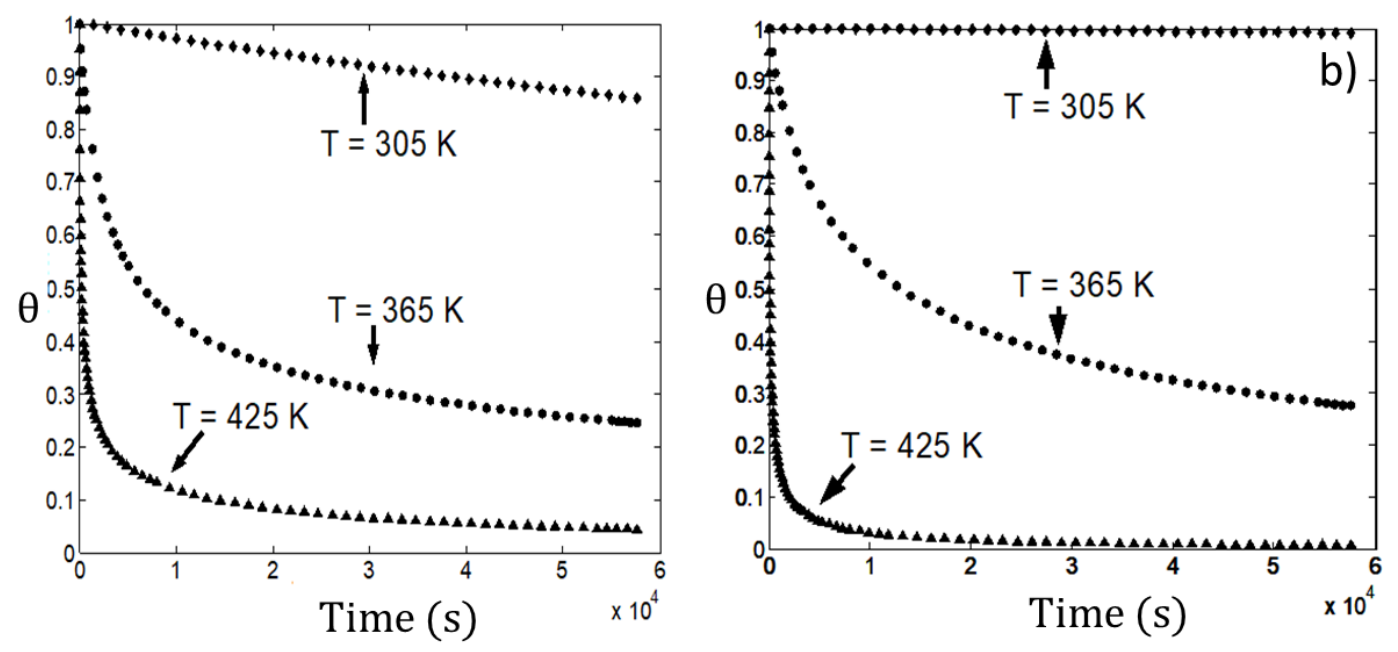

Figure 4: Surface coverage $(\theta)$ vs. time calculated using the optimized value of $E_{d}(\theta)$ at three different temperatures $(T=305,365$ and $425 \mathrm{~K})$ for the samples: a) Pt3-S and b) Co10-1S.

A more complex analysis is necessary for sample Co10-S2 where, due to Bartholomew's method ${ }^{\text {[22], }}$ it has been possible to record both the desorption of the two hydrogen species peaks (P1 and P2 respectively, see figure 1): one (P1) more weakly adsorbed, the other (P2) more strongly. Ernst et al ${ }^{[31]}$ have shown that on cobalt single crystal (1010) surface two types of hydrogen are chemisorbed ( $\alpha$ and $\beta$ ); also Dus and Lisowski ${ }^{[32]}$ have detected below $300 \mathrm{~K}$ two desorption states for hydrogen. 
The energy involved in the hydrogen reversibility (adsorption - desorption) is a function of support, dispersion, metal loading and extent of reduction to metal ${ }^{[22]}$. It is out of the aim of this work to deep the question if peak P1 of figure $1 \mathrm{~b}$ is due totally to weak dissociatively chemisorbed hydrogen or at least, partially, to a physisorbed species. We have elaborated the energetic analysis of such peak using $n=1$ and $n=2$ in the equation 1 . In the first case $(n=1)$ hydrogen has been considered totally physisorbed, while for $n=2$ is totally dissociated considered. The results for such two cases are reported in figures $5 \mathrm{a}$ and $5 \mathrm{~b}$ and $6 \mathrm{a}$ and $6 \mathrm{~b}$, while the peak $\mathrm{P} 2$ of the same sample is analysed as shown in figures $5 \mathrm{c}$ and $6 \mathrm{c}$. Moreover, the results concerning the desorption energies $E_{d}(\theta)$ both for peaks $\mathrm{P} 1$ and $\mathrm{P} 2$ are collated in table 1. 


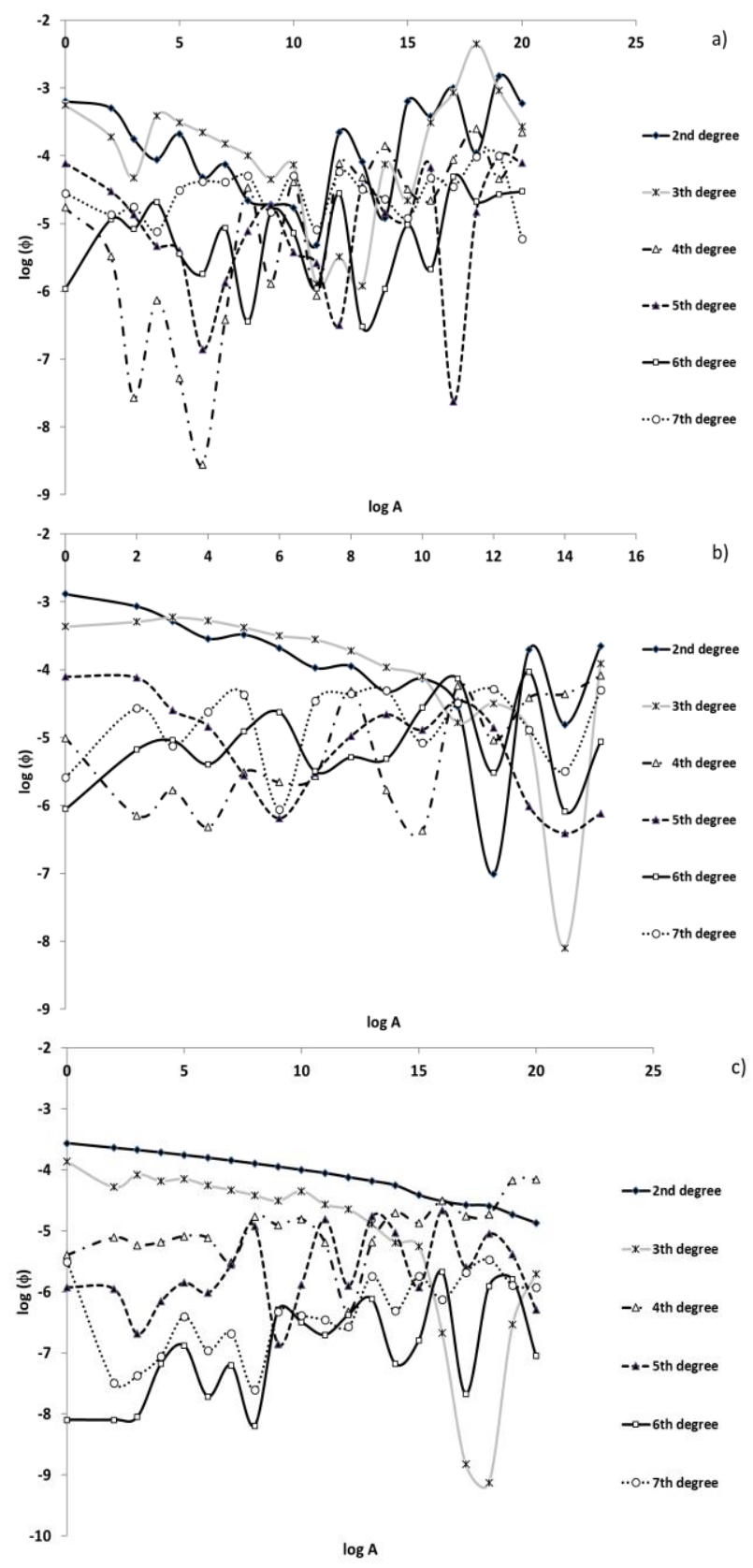

Figure 5: $\Phi$ function (equation 3) after the optimization procedure using different $A(\theta)$ values for the start of calculations and different $N$ degree (from 2 to 7 ) of equation (2) for Co10-S2 sample (a) TPD peak $A(n=1)$; (b) TPD peak $A(n=2)$; (c) TPD peak $B$ (chemisorbed hydrogen). Logarithmic scale. 

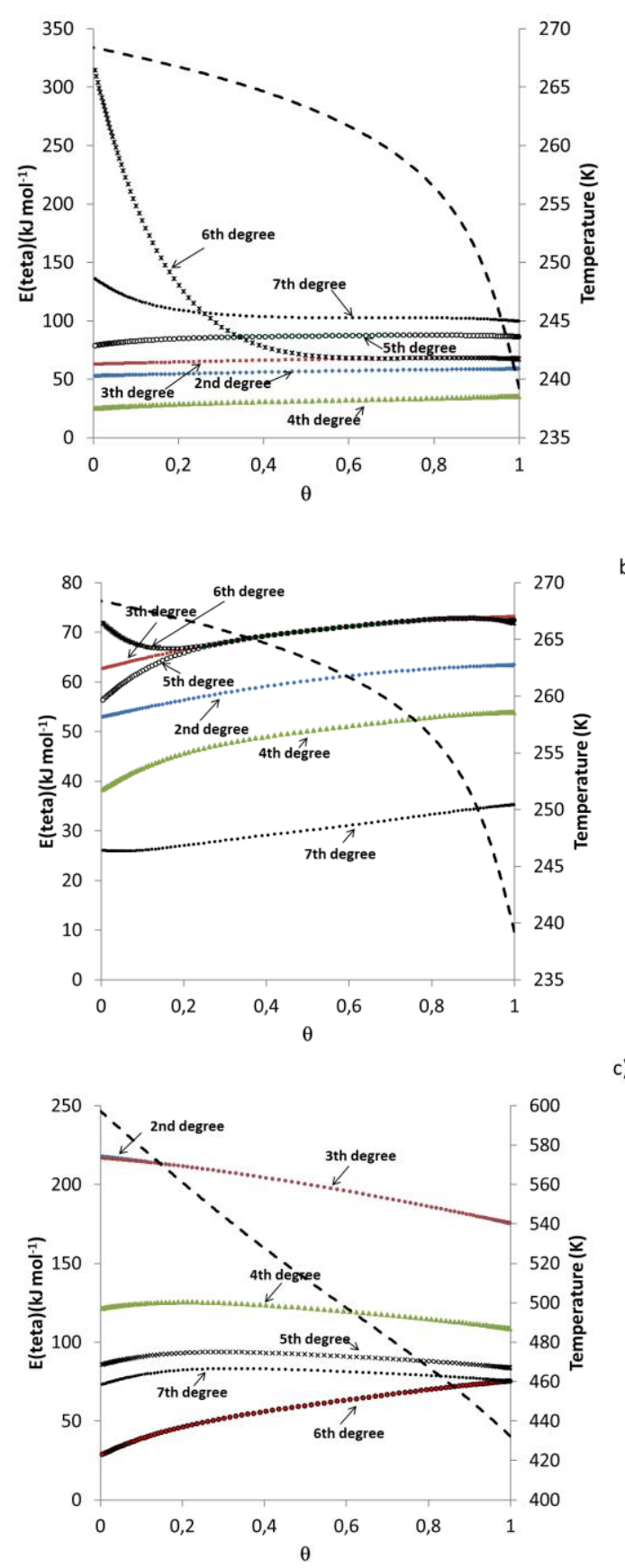

Figure 6: $E_{d}(\theta)$ calculated using the best optimized parameters (i.e. the parameters by which the lowest $\Phi$ function has been calculated) for each different degree $N$ of polynomial of equation (2) for ColO-S2 sample (a) TPD peak A $(n=1)$; (b) TPD peak $A(n=2)$; (c) TPD peak $B$ (chemisorbed hydrogen). Broken line on the secondary axis: temperature (K). 
The values of $E_{d}(\theta)$ for sample Co10-S2, having a $\mathrm{SiO}_{2}$ very similar to the one used by Zowtiak ${ }^{\text {[30] }}$ ranges (see table 1, sample Co10-2S, peak P2) from 175.7 to $216.8 \mathrm{~kJ} \mathrm{~mol}^{-1}$, which are in good agreement with the average Zowtiak's value of $168 \mathrm{~kJ} \mathrm{~mol}^{-1}$.

As for the peak $\mathrm{P} 1$ of the same sample it is very reasonable that both $E_{d}(\theta)$ values, calculate at $\theta=$ land $\theta=2$, are much lower than the previous reported data for P2 peak (see table 1). For systems where adsorbates are completely physisorbed (i.e. argon and nitrogen on hydroxylated and dehydroxylated silica) the isosteric heat of adsorption (at $\theta=0.5$ ) ranges from 8.8 to $13.4 \mathrm{~kJ} \mathrm{~mol}^{-1}$, roughly equal to activation energy of desorption ${ }^{[33]}$. The values of desorption energies obtained for hydrogen on sample Co10-2S when $n=1$ and $n=2$ for P1 peak (i.e. from 24.8 to $75 \mathrm{~kJ} \mathrm{~mol}^{-1}$, table 1), may indicate that the desorption energy can be attributed to a weakly chemisorption state of this adsorbate.

\section{Conclusions}

A new mathematical procedure is proposed in order to analyze TPD data to determine both the frequency factor and the distribution with the coverage $\theta$ of the activation energy of desorption based on the Polanyi-Wigner equation. In order to obtain meaningful parameters, it is suggested to explore large range of initial values for the frequency factor and different degrees (k) for the polynomial equation that correlate the desorption activation energy with the surface coverage $(\theta)$. Three different Pt and Co based catalysts have been considered as case studies. The results obtained by applying the mathematical elaboration are coherent with pre-exponential factor and desorption activation energies reported in literature for similar catalysts.

\section{Acknowledgments}

The work was partially financed by the call "Azione A, Piano sostegno alla ricerca - anno 2018 ” by Dipartimento di Chimica, Università degli Studi di Milano. 


\section{References}

[1] J. M. Kanervo, S. Kouva, K. J. Kanervo, R. Kolvenbach, A. Jentys, J. A. Lercher, Chem. Eng. Sci. 2015, 137, 807.

[2] C. Pirola, F. Galli, G. S. Patience, Can. J. Chem. Eng. 2018, 96, 2317.

[3] M. Niwa, N. Katada, Chem. Rec. 2013, 13, 432.

[4] J. Kanervo, T. Keskitalo, R. Slioor, A. Krause, J. Catal. 2006, 238, 382.

[5] K. C. Waugh, Chem. Eng. Res. Des. 2000, 78, 954.

[6] M. Schmal, D. V. Cesar, M. M. V. M. Souza, C. E. Guarido, Can. J. Chem. Eng. 2011, 89, 1166.

[7] S. Kouva, J. Kanervo, F. Schüßler, R. Olindo, J. A. Lercher, O. Krause, Chem. Eng. Sci. 2013, 89, 40 .

[8] W. Mao, J. Su, Z. Zhang, X.-C. Xu, W. Dai, D. Fu, J. Xu, X. Zhou, Y.-F. Han, Chem. Eng. Sci. 2015, 135, 312 .

[9] M. A. Salam, B. Abdullah, M. A. Islam, Chem. Eng. Res. Des. 2017, 118, 103.

[10] Y. Tokoro, J. Catal. 1979, 56, 110.

[11] A. K. Galwey, M. E. Brown, Thermochim. Acta 2002, 386, 91.

[12] M. Polanyi, E. Wigner, Zeitschrift f r Phys. 1925, 33, 429.

[13] J. L. Falconer, J. A. Schwarz, Catal. Rev. 1983, 25, 141.

[14] V. P. Bogillo, V. I.; Shkilev, J. Therm. Anal. Calorim. 1999, 55, 483.

[15] R. Luna, C. Millán, M. Domingo, C. Santonja, M. Á. Satorre, Vacuum 2015, 122, 154.

[16] R. Luna, M. Á. Satorre, C. Santonja, M. Domingo, Astron. Astrophys. 2014, 566, A27.

[17] A. Chakradhar, Y. Liu, J. Schmidt, E. Kadossov, U. Burghaus, Surf. Sci. 2011, 605, 1537.

[18] G. M. Muñoz Caro, A. Jiménez-Escobar, J. Á. Martín-Gago, C. Rogero, C. Atienza, S. Puertas, J. M. Sobrado, J. Torres-Redondo, Astron. Astrophys. 2010, 522, A108.

[19] G. Buzzi-Ferraris, F. Manenti, Chem. Eng. Sci. 2009, 64, 1061. 
[20] H. J. Motulsky, L. A. Ransnas, FASEB J. 1987, 1, 365.

[21] R. Giannantonio, V. Ragaini, P. Magni, J. Catal. 1994, 146, 103.

[22] R. D. Jones, C. H. Bartholomew, Appl. Catal. 1988, 39, 77.

[23] V. Ragaini, C. Pirola, S. Vitali, G. Bonura, C. Cannilla, F. Frusteri, Catal. Letters 2012, 142, 1452.

[24] C. Pirola, M. Scavini, F. Galli, S. Vitali, A. Comazzi, F. Manenti, P. Ghigna, Fuel 2014, 132, 62.

[25] P. Bianchi, C.L.M.; Martini, F.; Moggi, Catal. Letters 2001, 1, 65.

[26] M. Procop, J. Völter, Surf. Sci. 1972, 33, 69.

[27] W. Lisowski, Appl. Surf. Sci. 1988, 31, 451.

[28] R. J. Madix, G. Ertl, K. Christmann, Chem. Phys. Lett. 1979, 62, 38.

[29] J. J. Stephan, V. Ponec, W. M. H. Sachtler, Surf. Sci. 1975, 47, 403.

[30] J. Zowtiak, J. Catal. 1983, 83, 107.

[31] K. -H. Ernst, E. Schwarz, K. Christmann, J. Chem. Phys. 1994, 101, 5388.

[32] R. Duś, W. Lisowski, Surf. Sci. 1976, 61, 635.

[33] F. Faglioni, W. A. Goddard, J. Chem. Phys. 2005, 122, 014704. 